\title{
Polymerase chain reaction detection methods of Survival Motor Neuron genes- a review
}

\author{
Azra Alimanovićí ${ }^{*}$, Jasmin Šutković \\ ${ }^{1,2}$ Genetic and Bioengineering department, International University of Sarajevo
}

*Corresponding author: aalimanovic@ius.edu.ba
() The Author
2020.

Published by

ARDA.

\begin{abstract}
Two SMN (survival motor neuron) genes are presented in the human genome: SMN1, which present the telomeric gene whose homozygous deletion or mutation like gene conversion, causes spinal muscular atrophy (SMA), and SMN2, the centromeric version whose copy number modulates the phenotype of SMA These genes are commonly detected by Polymerase Chain reaction-based methods, and these are MLPA (Multiplex ligation-dependent probe amplification), qPCR (quantitative Polymerase chain reaction) and PCR-RFLP (Polymerase chain reaction-Restriction fragment length polymorphism). This paper reviews the current standing of the most common PCR methods used in the detection of spinal muscular atrophy genes. MLPA, qPCR, and PCR-RFLP currently represent the most common methods of choice for the detection of mutations, especially for deletion and duplication mutations.
\end{abstract}

Keywords: Mutations, homozygous deletion, phenotype, survival motor neuron, spinal muscular atrophy

\section{Introduction}

Heredity is the biological process by which traits are transmitted from parents to their offspring. Genetic trait can be dominant and recessive. Dominant genetic trait represents the affected individual who have two copies of mutant gene, while recessive genetic trait require that individual have one copy of mutant gene and one copy of normal gene. Spinal muscular atrophy is an autosomal recessive disease. The disease is characterized by degeneration of the anterior horn cells of the spinal cord and represent the second most common fatal autosomal recessive disease after cystic fibrosis [1]. SMA is disease of motor neuron and neuromuscular disease that affects the peripheral nervous system, including the body of the motor neuron (located inside the spinal cord), the axons of motor neuron (projections from the cell body to the muscles), the neuromuscular junctions (connection between the motor neuron axon and the muscle), or basically it can affect the muscles themselves [2]. Spinal muscular atrophy is characterized by muscle weakness and atrophy where an attack of weakness ranges from pre-birth to adulthood. Weakness is symmetrical, proximal, distal, and progressive [3].

\subsection{SMN mutations}

The main causes of the disease are mutations responsible for 5q-SMA which can be deletions, gene conversion and point mutations. Deletions include partial or complete removal of the SMN1 gene. In gene conversion, the SMN1 gene is "converted" to a gene similar to SMN2 because "C" in exon 7 changes to "T". In case with deletions and gene conversions, patients with SMA lack SMN1 exon 7, which is called 
homozygous absence of SMN1 exon 7, while the point mutations only affect a few nucleotides of the SMN1 gene and produce a non-functional and unstable SMN protein. Something that is very important to notice is that the patients with SMA have insufficient amounts of full-length SMN protein. Ninety-six percent of SMA patients display mutations for SMN1 gene, while 4\% are non-linked to 5q13. Of the 5q13-linked SMA patients, $96.4 \%$ show homozygous absence of SMN1 exons 7 and 8 or exon 7 only, whereas $3.6 \%$ present a heterozygosity with a subtle mutation on one chromosome and a deletion/gene conversion on the other chromosome. Among the 23 different subtle mutations, the Y272C missense mutation is the most frequent one, at $20 \%$. Only homozygous deletions or mutations of the SMN telomeric copy (SMN1) result in the SMA phenotype, and the levels of SMN expression driven by the centromeric copy (SMN2) in motor neurons inversely correlate with the severity of the disease. According to the literature, SMA due to SMN1 mutations has an incidence of approximately 1 in 10,000 newborns and a prevalence of approximately 1-2 per 100,000 persons $[4,5,6]$.

Clinical classification of SMA [7]

- $\quad$ Type 0 (Prenatal, congenital SMA)

- Type I (Werding Hoffmann disease, severe SMA)

- Type II (Moderate SMA)

- Type III (KugelbergWelanderova disease, mild SMA)

- $\quad$ Type IV (Adult SMA)

\subsection{SMN1 and SMN2 genes}

Survival motor neuron 1 gene (SMN1) is identified as a gene for determining SMA disease caused by the occurrence of homozygous absence including deletions or gene conversions (90\%), hybrid genomes (5\%) or by subtle disease $(<5 \%)$ [8]. This gene is located in a complex genomic structure with approximately $500 \mathrm{~kb}$ duplication on chromosome 5q13. The highly homologous copy of SMN1 is SMN2, which is described as an SMA modifier, and it is located in the central region of this locus. [9, 10] Both genes have been fully identified with some divergences that are mainly localized within 3'parts of these genes [11]. The survival motor neuron gene is located on chromosome 5, in the q12.2-q13.3 region, with two copies of homologues, SMN1 (telomeric copy) and SMN2 (centromeric copy), which differ by only eight nucleotides (five are introns and three are exons, located within exons 6, 7, and 8) [9, 11, 12, 13]. SMN1 can be distinguished from $S M N 2$ by two base changes in exons 7 and 8 . It means that the exon $7 \mathrm{C} \rightarrow \mathrm{T}$ transition is responsible for alternative splicing of exon 7 of the $S M N 2$ transcripts. Two genes generate different proportions of full-length transcripts: $S M N 1$ produces primarily full-length transcripts and a small amount $(\sim 10 \%)$ of transcripts lacking exon 5, whereas SMN2 produces primarily transcripts lacking exon 7 (40$50 \%$ ) plus minor amounts of full-length transcripts and transcripts lacking exon 5 or both exon 5 and exon 7 [14].

\section{Detection methods for SMN genes}

Polymerase chain reaction (PCR) method used in detection of SMN mutations, and they are: MLPA (Multiplex ligation-dependent probe amplification), qPCR (quantitative Polymerae chain reaction), and PCR-RFLP (Polymerase chain reaction-Restriction fragment length polymorphism).

\subsection{MLPA}

Multiplex ligation-dependent probe amplification (MLPA, developed by MRC Holland in 2002) is a versatile technique for relative quantification of different nucleic acid sequences in a single reaction, as can detect the copy number variation of genomic DNA sequences [15]. MLPA analysis is a high throughput analysis PCR based allowing up to 96 samples to be handled simultaneously, with results being available within $24 \mathrm{~h}$. The MLPA test for SMA molecular diagnosis is based on a kit containing several probes for 
the SMA critical region, including specific probes for SMN1 and SMN2 genes, probes that can hybridize both genes and other kit of probes for sequences mapped in critical SMA region (NAIP, GTF2H2, Ncadherin, CDH6 and RAD17 genes) or in other autosomal regions. Because of this specific set of assays, the MLPA test for the critical region of SMA can detect the copy number of both SMN1 and SMN2 genes, also both homozygous and heterozygous deletions of SMN1 or conversions to SMN2 can be detected, allowing diagnosis of affected patients or healthy carriers. Moreover, estimating the copy number of SMN2 can provide useful information for estimating the association of genotype and phenotype. Different groups have investigated the efficacy of MLPA in the molecular diagnosis of SMA, both in affected patients and in healthy controls $[16,17,18,19,20]$. The MLPA technique greatly improves diagnostics in SMA. Multiplex ligation-dependent probe amplification analysis, which is different from PCR-RFLP and allelespecific PCR (which only detect the absence of SMN1), detects the mechanism responsible for SMN1 loss (deletion or conversion to SMN2). Evaluation of the SMN2 copy number is also useful for establishing genotype-phenotype correlation in patients with SMA based on the evidence that the SMN2 copy number relates to severity of the disease [21].

The effect of MLPA technique in the molecular diagnosis of spinal muscular atrophy (SMA) is investigated by Zeng $J$ and his colleague [22] performed the experiment where peripheral blood samples were taken from 13 patients with SMA, 31 parents of SMA patients, 50 healthy individuals without family history of SMA, and 10 specimens of amniotic fluid from these families were collected too. Genomic DNA was analyzed by MLPA, conventional PCR-RFLP, and allele-specific PCR. These results are in line with a study conducted with conventional PCR-RFLP and allele-specific PCR. MLPA analysis showed that all of the 13 patients had homozygous deletion of the survival of motor neuron 1 (SMN1) gene, and there was a significant difference between the SMA severity (type I to type III) and SMN2 copy number $(\mathrm{P}<0.05)$. Out of 31 parents $29(93.5 \%)$ had 1 copy of SMN1, $2(6.5 \%)$ had 2 copies of SMN1. Among the 50 healthy individuals, 1 (2.0\%) had 1 copy of SMN1, 48 (96.0\%) had 2 copies of SMN1, and 1 (2.0\%) had 3 copies. The SMN1 copy number of the parents was significantly higher than that of the healthy individuals $(\mathrm{P}<0.01)$. Two of the 10 fetuses had homozygous deletion of SMN1.

As it mentioned previously that SMA disease is classified in V types, at which the type III is one of the milder and rarer forms of SMA. Gamze Bora-Tatar and his colleagues in their study [23] reported the molecular genetic characteristics of 24 Turkish patients with type III of SMA. Homozygous loss of SMN1 exons 7 and 8 was analyzed by PCR-RFLP and MLPA. SMN2, the homologue of SMN1 gene, and the neuronal apoptosis inhibitor (NAIP) gene were also evaluated for their effect on disease severity. They found that men born in related families predominated in the group, and these patients generally carried a homozygous loss of SMN1 exons 7 and 8 and four copies of the SMN2 gene without NAIP deletions. Based on the literature information so far stated, we can clearly conclude that the MLPA method has proven its accuracy in the diagnosis of SMA.

\section{2. qPCR}

qPCR refers to a biotechnology technique which is a powerful tool for detection, characterization and quantification of nucleic acids, as for analysis and quantification of gene expression [24].

It uses the RNA molecule as its template. However, for the final amplification the target gene, the RNA template needs to be converted into a complementary DNA (cDNA) molecule, known as reverse transcriptase polymerase chain reaction (RT-PCR) procedure. If compared to a simple PCR method, in qPCR method, the gene expression can be visualized in "real-time" using a computer software, with no need of additional quantification and verification methods [25].

Multiplex qPCR requires the use of probe-based assays, in which each probe is labeled with a unique fluorescent dye, resulting in different observed colors for each assay. One of the main and popular dye used 
in qPCR is SYBR Green and it is important for detection DNA amplification. The signal from each dye is used to quantifies the amount of each target separately in the same tube or well [26].

However, success of qPCR technique depends on the optimal primers which are used. Some of the considerations for primer design are the following: GC content, primer self-dimer, or secondary structure formation [27]. Few studies have presented this method as an effective tool to diagnose the SMN gene mutations. In study conducted in French the authors analyze the expression of SMN1 and SMN2 genes in 600 patients with sporadic ALS and 621 controls using a quantitative PCR method (qPCR). The authors found an association of ALS with an abnormal copy number, and they did not find the association with SMN2 copy numbers and there is no effect of SMN2 copy on the duration of evolution in ALS independently of SMN1 copy number. Regard that the abnormal SMN1 gene copy numbers are a genetic risk factor in sporadic amyotrophic lateral sclerosis. There was no modulator effect of the SMN2 [28]. Further, in study conducted in USA, between the January 2016 to January 2017, a multiplex TaqMan realtime quantitative polymerase chain reaction assay was effectively used for the screening of spinal muscular atrophy. They screened 3,826 newborns at three hospitals in New York City and tested newborns for the deletion in exon 7 of SMN1 [29]. Tae-Mi Lee et al. developed a reliable quantitative real-time PCR with SYBR green and studied 13 SMA patients with 24 parents, as well as 326 healthy normal individuals. The copy number of the SMN1 gene was determined by the comparative threshold $(\mathrm{Ct})$ method, and albumin was used as the reference gene. The ratio of homozygous deletions of SMN1 patients was 0.00, and the ratio of hemizygous deletions of SMN1 parents ranged from 0.39 to 0.59 . The delta-delta Ct ratio of 7 individuals among 326 normal individuals was within the carrier range, $0.41-0.57$. According to these data, they estimated the carrier and prevalence of SMA at 1/47 and 1/8 496, respectively, in the Korean population [29]. These data showed that there will be no large differences in the prevalence of SMA compared to Western countries. Because the prevalence of SMA is higher than other autosomal recessive disorders, the real-time PCR (qPCR) carrier detection method may be a useful genetic counseling tool [30].

\subsection{PCR-RFLP}

PCR-RFLP method, also known as CAPS (Cleaved amplified polymorphic sequence) is a popular molecular genetic detection method. The standard molecular diagnosis of SMA is based on a PCR-RFLP assay that can detect homozygous loss of SMN1 gene [31]. This method includes the PCR amplicon treated by specific RE (restriction enzyme) that cleaves DNA at a single restriction site, known as recognition site, to generate several DNA fragments into different sizes. The digested amplicons are then loaded into a gel and applied to an electric field. High concentration of amplicons is required to perform successful PCRRFLP. Technique is usually performed on horizontal agarose gel system [32]. The advantage of PCR-RFLP in comparison to allele-specific PCR method, is that PCR-RFLP identifies the SMN1 exon 7 by amplifying $10 \mathrm{pg}$ of genomic DNA, and could differentiate SMN1 from SMN2 at the 100-pg DNA level (Dra I digested SMN2 fragments served as an internal control for PCR efficiency) [33]. Scientist Yu-Wei Jin and his colleagues explored the applicability and limitation of PCR-restriction fragment length polymorphism (PCR-RFLP) method, for genetic diagnosis of spinal muscular atrophy (SMA). In this study, PCR-RFLP was applied to detect potential deletion in exons 7 and 8 of SMN1 gene in 935 suspected cases with SMA. Multiplex ligation-dependent probe amplification (MLPA) was carried out to analyze dosage alteration of SMN1 gene in 339 of such cases. To confirm the accuracy of PCRRFLP method for homozygous and heterozygous deletions detection, the consistency of PCR-RFLP and MLPA results were assessed with a Pearson Chi-square test.The result showed that homozygous deletion of exon 7 of SMN1 was detected in 590 suspected cases. The rate of diagnosis was therefore $63.1 \%$ (590/935). For the 339 suspected cases, PCR-RFLP and MLPA respectively identified 194 and 196 homozygous deletions in the exon 7 of SMN1 gene, suggesting a good consistency $(98.9 \%)($ Chi-square $=$ $0.2, \mathrm{P}=0.88$ ). However, only 4 of 339 cases was found to carry a heterozygous deletion of SMN1 exon 7 
by PCR-RFLP, in contrast with 17 detected by MLPA. The consistency only reached $23.5 \%$, for which statistical significance was detected (Chi-square $=8.29, \mathrm{P}<0.01)$ [34]. Yu- hua Liang and his colleague from China, analyze the deletion of SMNI and NAIP genes in southern Chinese children with SMA. PCR-RFLP was performed to detect the deletion of both exon 7 and exon 8 of SMNI and exon 5 of NAIP, in 62 southern Chinese children with strongly suspected clinical symptoms of SMA.The findings of homozygous deletions of exon 7 and/or exon 8 of SMN1 gene confirmed the diagnosis of SMA, and suggested that the deletion of SMN1 exon 7 is a major cause of SMA in southern Chinese children, and that the NAIP gene may be a modifying factor for disease severity of SMA1. The molecular diagnosis system based on PCRRFLP analysis can conveniently be applied in the clinical testing, genetic counseling, prenatal diagnosis and preimplantation genetic diagnosis of SMA [35].

\section{Conclusion}

The survival motor neuron (SMN) is gene which causes the disease called spinal muscular atrophy in humans. SMA is an autosomal recessive disease characterized by progressive degeneration of lower motor neurons. SMA is clinically classified in five types (prenatal, severe, moderate, mild and adult SMA), based on clinical onset of the disease. There are two copies of SMN gene, one is centromeric and other is telomeric, called SMN1 and SMN2 gene. All SMN gene mutations are detected by different PCR methods: MLPA, qPCR, PCR-RFLP. The most common used method is MLPA, where the one of the most diagnostic method for analysis SMA deletion is RT-PCR, qPCR (Taq Man, Sybr Green, FRET). For an accurate genetic diagnosis and inclusion into the registry, results of non - quantitative method (PCR-RFLP) should be used and confirmed by quantitative analysis.

\section{References}

[1] Lydie Bürglen, Suzie Lefebvre,OlivierClermont,Philippe Burleta,Louis Viollet,Corinne Cruaud,Arnold Munnich,Judith Melki. Structure and Organization of the Human Survival Motor Neurone (SMN) Gene. ; Received 19 September 1995, Accepted 28 December 1995, Available online 25 May 2020.

[2] Simard R Louise. The Genetics of Spinal Muscular Atrophy. 2009 November: p. 3-16

[3] Kiernan, Michelle A. Farrar and Matthew C. The Genetics of Spinal Muscular Atrophy: Progress and Challenges.2015 Apr; 12(2): 290-302. Published online 2014 Nov 21

[4] B.Wirth. An update of the mutation spectrum of the survival motor neuron gene (SMN1). Human Mutation. 2000; 15(3):228-37

[5] LivioPellizzoni, Bernard Charroux, and Gideon Dreyfuss. SMN mutants of spinal muscular atrophy patients are defective in binding to snRNP proteins. September 28, 199996 (20) 11167-11172

[6] Ingrid E.C.Verhaart, Agata Robertson, Rebecca Leary, Grace McMacken, Kirsten Konig,Janbernd Kirschner, Cynthia C.Jones, Suzanne F.Cook,Hanns Lochmuller.

A multi-source approach to determine SMA incidence and research ready population. Neurology. 2017; 264;1465-1473

[7] Basil T. Darras . Spinal muscular atrophies. Ped Clin North Am. 2015; 62, 743-766

[8] S Lefebvre, L Bürglen, S Reboullet, O Clermont, P Burlet, L Viollet, B Benichou, C Cruaud, P Millasseau, M Zeviani, et al. Identification and Characterization of a Spinal Muscular AtrophyDetermining Gene. Cell. 1995 January; 80(1):155-65

[9] Thomas W Prior, Meganne E Leach,Erika Finanger. Spinal Muscular Atrophy. Gene Reviewes. 19932020

[10] Alias L, Bernal S, Fuentes-Prior P, Barcelo M, Also E, Martines-Hernandez R, et al. Mutation update of spinal muscular atrophy in Spain: molecular characterization of 745 unrelated patients and identification of four novel mutations in the SMN1 gene. Human Genetics. 2009; 125:29-39

[11] Calucho M, Bernal S, Alias L,March F,vencesla A,Rodriguez-Alvarez FJ,et al. Correlation between SMA type and SMN2 copy number revisited: an analysis of 625 unrelated 
Spanish patients unrelated Spanish patients and a compilation of 2,834 reported cases. Neuromuscular Disorders. 2018; 28:208-15

[12] Bürglen L, Lefebre S,Clermont O,Burlet P,Viollet L,Cruaud C,et al. Structure and organization of the human survival motor neurone [SMN] gene. Genomics.1996; 32:479-82 Biros I.Forest .

Spinal muscular atrophy:Untangling the knot? J Med genet. 1999; 36:1-8

[13] Tiziana Vitali, Vittorio Sossi, Francesco Tiziano, Stefania Zappata, Anna Giuli, Maria ParavatouPetsota. Detection of the Survival Motor Neuron (SMN) Genes by FISH: Further Evidence for a Role for SMN2 in the Modulation of Disease Severity in SMA Patients. Human Molecular Genetics. 1999 December; 8(13, Pages 2525-2532)

[14] Nadia Passon, Giorgia Dubsky de Wittenau, Irena Jurman, Slobodanka Radovic, Elisa Bregant, Cristiano Molinis, Giuseppe Damante, Incoronata Renata Lonigro. Quick MLPA test for quantification of SMN1 and SMN2 copy number. Mol Cell Probes. 2010 Oct; 24(5):310-4

[15] Arkblad E.L., Darin N., Berg K., Kimber E., Brandberg G., Lindberg C., Holmberg E., Tulinius M., Nordling M. Multiplex ligation-dependent probe amplification improves diagnostics in spinal muscular atrophy. Neuromuscular Disord. 2006; 16:830-838

[16] Scarciolla O., Stuppia L., Angelis M.V., Murru S., Palka C., Giuliani R., Pace M., Muzio A., Torrente I., Morella A., et al. Spinal muscular atrophy genotyping by gene dosage using multiple ligation-dependent probe amplification.. Neurogenetics. 2006; 7:269-276

[17] Huang C.H., Chang Y.Y., Chen C,H., Kuo Y.S., Hwu W.L., Gerdes T., Ko T.M. 9:241-248. Copy number analysis of survival motor neuron genes by multiplex ligation-dependent probe amplification. Genet. Med. 2017; 9:241-248

[18] Arkblad E., Tulinius M., Kroksmark A.K., Henricsson M., Darin N. 2009; 98:865-872. A population-based study of genotypic and phenotypic variability in children with spinal muscular atrophy. Acta Paediatr.. 2009; 98:865-872

[19] Yoon S., Lee K.A. Determination of SMN1 and SMN2 copy numbers in a Korean population using multiplex ligation-dependent probe amplification. Korean J Lab Med. 2010 February; 30(1):93-96

[20] Arkblad E., Tulinius M., Kroksmark A.K., Henricsson M., Darin N. 2009; 98:865-872.

A population-based study of genotypic and phenotypic variability in children with spinal muscular atrophy. Acta Paediatr.. 2009; 98:865-872

[21] Zeng J, Penghui Zhang, Xiaoyan He, Shan Liu, Shi Tang, et al. Molecular diagnosis of spinal muscular atrophy by multiplex ligation-dependent probe amplification. Chinese.2008 December; 88(46):3262-4

[22] Bora Tatar G, Yesbek Kaymaz A, Bekircan Kurt CE, Erdem Ozdamar S, Erdem Yurter H. Spinal muscular atrophy type III:Molecular genetic characterization of Turkish patients.

Eur J Med Genet. 2015 December; 58(12):654-8

[23] Petr Kralik and Matteo Ricchi. A Basic Guide to Real Time PCR in Microbial Diagnostics: Definitions, Parameters, and Everything. Front Microbiol. 2017;8:108. Published online 2017 Feb 2

[24] Steve F, Hawkins Paul C. Multiplex Analyses Using Real-Time Quantitative PCR. Methods Mol Bio. 2017; 125-133

[25] Brenda Thornton, Chhandak Basu. Rapid and simple method of qPCR primer design. Methods Molecular Biology. 2015; 1275:173-179

[26] P.Corcia, W Camu, J-M Halimi, P Vourc'h, C Antar, S Vedrine, B Giraudeau, B de Toffol, C R Andres, French ALS Study Group. Smn1 gene,but not SMN2, is a risk factor for sporadic ALS. Neurology. 2016 Oct 10; 67(7):1147-50

[27] Jennifer N Kraszewski, Denise M Kay, Colleen F Stevens, Carrie Koval, Bianca Haser, Veronica Ortiz, Anthony Albertorio, Lilian L Cohen, Ritu Jain, Sarah P Andrew, Sally Dunaway Young, Nicole M LaMarca, Darryl C De Vivo, Michele Caggana, Wendy K Chung. Pilot study of populationbased newborn screening for spinal muscular atropfy in New york state. Genet Med. 2018 June; 20(6)(608-613)

[28] Jennifer N Kraszewski MPH, Denise M Kay PhD, Colleen F Stevens PhD, Carrie Koval MS, Bianca Haser BS, Veronica Ortiz MHS, Anthony Albertorio BA, et al.

Pilot study of population-based newborn screening for spinal muscular atrophy in New York state. Genetics in Medicine volume 20, pages 608-613 (2018). Published:12 October 2017

[29] Tae-Mi Lee, Sang-Wun Kim, Kwang-Soo Lee, Hyun-Seok Jin, Soo Kyung Koo ,Inho Jo, Seongman Kang, Sung-Chul Jung. Quantitative Analysis of SMN1 Gene and Estimation of SMN1 Deletion 
Carrier Frequency in Korean Population Based on Real-Time PCR. Korean. 2004 December; 19(6):870-3

[30] Junhua Xiao, Xiujuan Xin, Xiaohui Luan, Dongzhi Wei,Shengli Yang. A modified simple RFLPPCR method for single nucleotide polymorphism (SNP) typing. Genet. Mol. Biol. vol.29 no.3 Sao Paulo, 2006

[31] Establishment of a Molecular Diagnostic System for Spinal Muscular Atrophy Experience From a Clinical Laboratory in China Jian Zeng, Yanhong Lin, Aizhen Yan, LongfengKe, Zhongyong Zhu, and Fenghua Lan. The Journal of Molecular Diagnostics, Vol. 13, No. 1, January 2011

[32] Hayder O. Hashim, Mohammed Baqur S.Al Shuhaib. Exploring the Potential and Limitations of PCR- RFLP and PCR-SSCP for SNP Detection: A Review. Applied Biotechnology Reports. 2019 December; 6(4):137-144

[33] Ruliang Xu, Shuji Ogino, Va Lip, Hong Fang, Bai-Lin Wu. Comparison of PCR-RFLP with allelespecific PCR in genetic testing for spinal muscular atrophy. Genet Test Winter. 2003; 277-281

[34] Yu-wei Jin, Yu-jin Qu, Hong Wang, Jin-li Bai, Fang Song. Limitation of PCR-RFLP method for the detection of genetic mutations in spinal muscular atrophy. $2012 \mathrm{Feb} ; 29(1): 34-7$

[35] Yu-hua Liang, Xiao-ling Chen, Zhong-sheng Yu, Chun-yue Chen, Sheng Bi, Lian-gen Mao, Bo-lin Zhou, Xian-ning Zhang. Deletion analysis of SMN1 and NAIP genes in Southern Chinese children with spinal muscular atrophy. J Zhejiang Univ Sci B. 2009 Jan;10(1):29-34 